\title{
Delayed and Unusual Presentation of Impacted Laryngeal Foreign Body in Adult
}

YB Ngamdu, MB Sandabe, AM Kodiya, A Isa, HI Garandawa

Department of ENT Surgery, University of Maiduguri/University of

Maiduguri Teaching Hospital, Maiduguri, Nigeria.

Abstract:

Laryngeal foreign body is very rare in sane adults. Diagnosis is mostly achieved by high index of suspicion and clinico-radiological examinations. Delayed presentation pose a high risk of complications and also presents a challenge to both otolaryngologists and anaesthiologists. Prompt intervention is all that is needed to avoid morbidity and/or mortality. We report a case of delayed and unusual presentation of impacted laryngeal foreign body in a 35 year old man presenting with unremitting hoarseness for 3 months.

Key words: Larynx, Foreign Bodies, Tracheostomy, Hoarseness, Aspiration.

\section{Introduction}

Laryngeal impaction of foreign bodies are very rare as most aspirated foreign bodies passes through the laryngeal inlet and get lodged lower down in the airway or is coughed out by cough reflex. If foreign body lodges at the glottis area, morbidity and mortality are high, due to their potential for sudden airway obstruction. Delayed presentation or delayed diagnosis has a high risk of complications including death. This presents a continuous challenge to the otolaryngologists and anaesthiologist especially in paediatric age. Narrow laryngeal air column in children causes hindrance to intubation and ventilation and, moreover laryngeal oedema develops quickly by the trauma and/or the tissue reaction caused by foreign body. We report a case of delayed presentation of impacted laryngeal foreign body (paracetamol blister) in a 35 year civil servant who presented with three months history of hoarseness.

\section{Case Report}

A 35 year old male civil servant presented to our clinic with 3 months history of unremitting hoarseness. He had occasional bouts of cough. No preceding history of trauma to the neck or upper respiratory tract infections was elicited. He denied history of aspiration of foreign body. No history of stridor, difficulty with breathing or dysphagia, psychiatric tendency or family history of psychiatric disorders was elicited. He neither smoke nor drank alcohol. $\mathrm{He}$ was taking orally well and slept fairly well. Noting specific was noted in clinical examination of chest and abdomen. Chest $\mathrm{X}$-ray done at this time was reported normal.

Corresponding Author: Dr. YB Ngamdu

Email: ybugam@yahoo.com

Received: October 4, 2013 | Accepted: January 8, 2014 | Published Online: February 15, 2014

This is an Open Access article distributed under the terms of the Creative Commons Attribution License (creativecommons.org/licenses/by/3.0)

Conflict of interest: None declared | Source of funding: Nil | DOl: http://dx.doi.org/10.17659/01.2014.0017 
Flexible fibre-optic nasophayngolaryngoscopy revealed a whitish plastic material with sharp edge pointing upward impacted at the glottis area, covered with mucoid secretion. X-Ray soft tissue neck, lateral view showed a ring like opaque shadow with central lucency [Fig.1].

He was prepared for elective tracheostomy; direct laryngoscopy and foreign body removal under general anaesthesia. Findings were a cut blister of paracetamol tablets [Fig.2] impacted vertical to the long axis of the glottis more to anterior commissure, with inflammation of both true vocal cords. Subsequent to removal of blister, patient did well. He was started on 5 days course of antibiotic and anti-inflammatory drugs.

Post-operative period was uneventful. Postoperative X-ray soft tissue of the neck done on the $5^{\text {th }}$ day showed adequate laryngeal column, subsequently decanulation process was commenced. He tolerated the decanulation and he was discharge on the $6^{\text {th }}$ post-operative day. During the fellow up period, he did well; all the symptoms he presented with on the first visit were resolved.

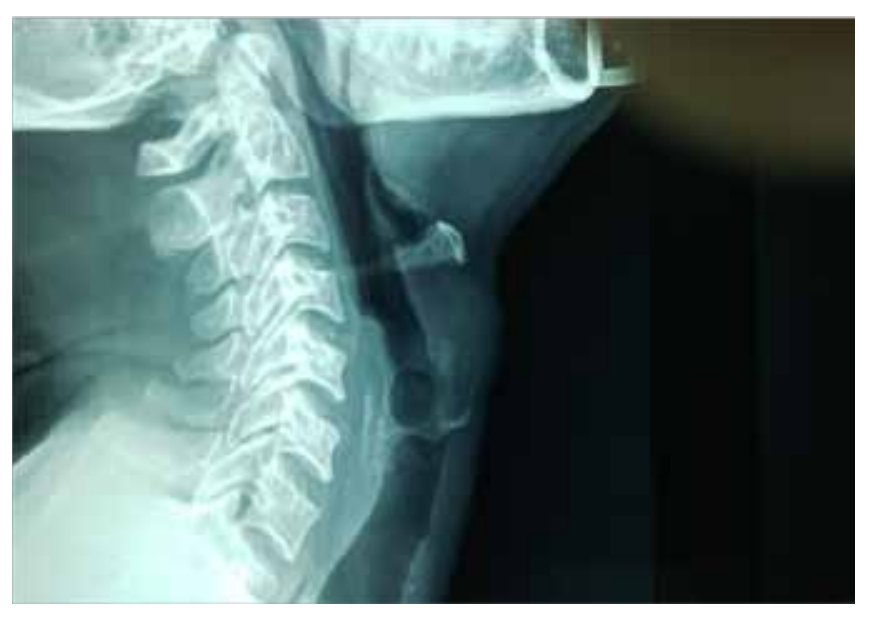

Fig.1: Lateral view soft tissue $X$-ray of the neck showing a ring like opaque with central lucency at the laryngeal level.

\section{Discussion}

A foreign body is defined as an object or substance foreign to the location where it is found. Foreign body in the aero-digestive tract are commonly found in paediatric age (up to 3-4 years) than in adults, this is because children in this age group are curious in nature, have strong oral tendency and lacks molar teeth [1-3].

Larynx is not a common site of foreign body impaction since a foreign body lodged at this site is either coughed out or moves down into the tracheobronchial tree [4]. The most common site of foreign body is bronchi (83\%) followed by trachea $(12 \%)$ and larynx (2.9\%) [1]. The larynx being an uncommon site, can be explained by protection of the airway by the laryngeal sphincters and cough reflexes. Foreign body aspiration in the larynx occurs more frequently in female with female:male ratio 2:1 [5]. Adults who are unable to protect the airway such as person with mental retardation, alcoholism, psychosis and neurological disorder are commonly affected [2]. None of these were found in the index case. It has reported that up to $5-50 \%$ of patients with foreign body aspiration does not have a contributory history available [2]. $67 \%$ complications occur due to delay in presentation and diagnosis. When the removal was delayed for

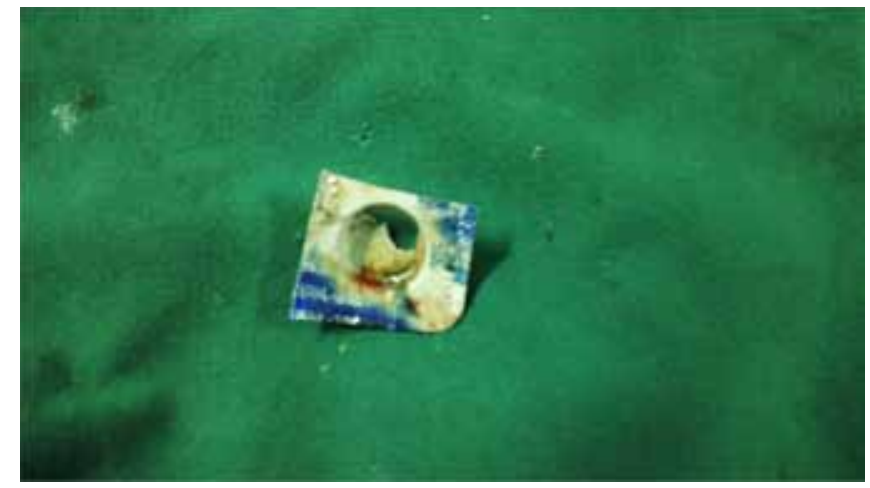

Fig.2: A blister of paracetamol tablet removed from the glottis of a 35 year old man. 
more than 24 hours, $62 \%$ experience associated complications [1].

A various nature of foreign bodies have been reported in literature ranging from nuts, seeds, teeth, erasers, pencils, safety pins, whistles, tracheostomy tubes, fish bones, chicken bones, stones, earrings, plastic toys, are most common but no report of drug protector in sane adult [2,3,5]. Some organic foreign body cause surrounding tissue reaction and this may result in a condition known as Arachidic bronchitis. This is seen radiologically as a spidery pattern, on chest radiographs [1].

The foreign body lodgement in the airway can lead to partial or complete airway obstruction. Partial obstruction occurs when the upper airway is partially occluded or if the obstruction occurs distal to carina. Patients may present weeks to month after aspiration. Complete airway obstruction occurs in the upper airway at level above the carina and can lead acute respiratory distress. This would lead to death if the foreign body is not dislodged or removed immediately.

The symptoms and severity of foreign body aspiration depends on the size, site, composition and duration for which the foreign body is present in situ. The natural history of foreign body aspiration follows these phases: (i) Initial phase of choking, gasping, and coughing or airway obstruction. (ii) Asymptomatic phase resulting in reduction or cessation of symptoms, lasting hours, days to weeks.

(iii) Complication phase producing erosion or progressive obstruction leading to pneumonia, lung abscess or atelectasis. The laryngeal foreign body patients present with hoarseness, aphonia, stridor, difficulty with breathing, haemoptysis, subjective feeling of the foreign body following history of foreign body especially in sense adults, the cough paroxysm are seen in 59\% of patients [1]. On rare occasion, hoarseness may be the sole symptom of foreign body aspiration as also seen in our case [6].
Diagnostic imaging is vital in case of foreign body aspiration. High kilovoltage anterior-posterior (in paediatric age) and posterior-anterior (in adults) and, lateral imaging of the soft tissue neck, inspiratory and expiratory films are commonly used. This produces greater definition of the airway, which decreasing the effects of the surrounding tissue [1]. Radio opaque foreign bodies are easy to diagnose by using radiographs. Imaging studies have a sensitivity of $73 \%$ and specificity of $45 \%$ [7]. If findings are negative for foreign body and clinical suspicion still remain high, laryngoscopy and/or bronchoscopy should be performed in operating theatre.

The treatment of foreign body in the airway is to remove the foreign body and it poses a greater challenge to the surgeon and anaesthiologist because they present as medical emergency and any delay in securing the airway would results in catastrophe. Once the diagnosis is done or any suspicion is present, intervention should be instituted. This depends on the severity, availability of facilities and expertise in the environment where you are working. The recommendations to be followed for foreign bodies lodging in larynx include head down back blow manoeuvre for infants. In toddlers, chest thrust manoeuvre, using sternal compression to increase intrathoracic pressure, in an effort to expel the foreign body from the airway is used.The Heimlich manoeuvre reserved for older children or adults is done by placing your arms around patient's chest and giving abdominal thrust $[2,8]$.

The above procedures are attempted as a first means and performed only in case of complete obstruction. It is contraindicated in partial obstruction, for fear of causing complete (total) obstruction [8]. Cricothyroidotomy or emergency tracheostomy should be done in emergency room by a doctor or person trained in this field in cases of complete airway obstruction and the above mentioned manoeuvre failed. Airway foreign 
bodies are removed mostly safely under general anaesthesia. The most common approach has been an inhalation induction and direct laryngoscopy or bronchoscopy during spontaneous ventilation, though there is a paucity of published evidence that this is superior to an intravenous induction and controlled ventilation. A wide variety of tricks are available for managing different airway foreign bodies [8].

Impacted laryngeal foreign body poses a greater challenge to both otolaryngologist and anaesthiologist in that they seem so easily accessible that in haste to remove them under short apnoeic technique or endotracheal intubation, one may push them further inside resulting in worsening the situation. Emergency/elective tracheostomy (as in our own case) should be performed prior to any attempt at removal since this would perform dual function of securing the upper airway as well as helping in preventing any post-operative catastrophe due to exacerbation of the already existing oedema of the glottis and subglottic area following manipulation during the removal of the foreign body [5]. It is also helpful in reviewing the larynx for second foreign body or complications [8]. The foreign bodies should never be removed without first confirming and documenting its presence and then making sure that one has proper illumination, instrumentation and expertise to successfully remove the foreign body. A lack of these and under haste may sometimes prove to be fatal [5]. Normal radiograph does not rule out foreign body, be ready and equipped, do not make a close case open, do not turn a non-obstructing foreign body into obstructing one and do not miss the second foreign body.

\section{References}

1. Diop EM, Tall A, Diout R. Laryngeal foreign body: management in Children in Senegal. Arch Paediatric. 2000;7:10-15.

2. Ellan M. Tracheo-bronchial foreign bodies. Otolaryngologic Clinic of North America. 2000;33:179-185.

3. Bienzdo RE, Walter SM. Foreign body aspiration through tracheostomy. Brazillian Journal of Oto Rhino Laryngol. 2005;71:234-236.

4. Hathiram BT, Khatter V, Hiwarkar S, Rai R. An unusual presentation of impacted foreign body in the Adult larynx. Indian J Otolaryngol Head Neck Surg. 2011 ;63:96-98.

5. Kumar GK, Ganesan C, Pilai SA, Radhakrishnan E. A subglottic foreign body in a child. Indian J Anaesth. 1997;41:207-208.

6. Hanukoglu. Loss of voice as sole symptom of subglottic foreign body aspiration. Am J Dis Child. 1986; 140:973.

7. Silva $A B$, Muntz $H R$, Clary R. Utility of conventional radiography in the diagnosis and management of paediatric airway of foreign bodies. Annals of Otology Rhinology and Laryngology. 1998;107:834-838.

8. Kansara AH, Shah HV, Patel MA, Manjunatharao SV. Unusual case of laryngeal foreign body. Indian J Otolaryngol Head Neck Surg. 2006;59:63-65. 